\title{
Hyperfine Populations Prior to Muon Capture.
}

\author{
J.G.Congleton \\ Instituut Voor Theoretische Fysica, Universiteit te Utrecht, \\ Postbus 80.006, 3508 TA Utrecht, The Netherlands.
}

\begin{abstract}
It is shown that the $1 \mathrm{~S}$ level hyperfine populations prior to muon capture will be statistical when either target or beam are unpolarised independent of the atomic level at which the hyperfine interaction becomes appreciable. This assertion holds in the absence of magnetic transitions during the cascade and is true because of minimal polarisation after atomic capture and selective feeding during the cascade.
\end{abstract}

PACS numbers: 23.40, 36.10

Typeset Using REVTEX 
The muon capture rate by a nucleus from the $1 \mathrm{~S}$ atomic level, depends on the populations of the hyperfine states $f, f_{z}$. The isotropic rate depends only on the total populations of the two hyperfine levels $f^{ \pm}=i \pm \frac{1}{2}$, where $i$ is the total angular momentum of the nucleus and is non-zero. In this letter, it is shown that these populations are statistical independent of the atomic level at which the hyperfine interaction becomes appreciable, provided at least one of the target nucleus or the muon beam is unpolarised. This assertion holds in the absence of magnetic transitions during the atomic cascade.

Mukhopadhyay [1] has shown that the populations are statistical when the hyperfine interaction acts only at the $1 \mathrm{~S}$ state and at least one of the target nucleus or beam is unpolarised. Measurement of residual muon polarisations in light nuclei [2] show that the hyperfine interaction must be active at atomic states higher than $n=1$. The question arises as to whether the populations remain statistical when the hyperfine interaction acts before the muon reaches the $1 \mathrm{~S}$ state.

This question is of current interest due to the experimental proposal [3] to measure the statistical capture rate by ${ }^{3} \mathrm{He}$ to a precision of $1 \%$ at PSI. A deviation of $2.5 \%$ from statisticity in the hyperfine populations would change the measured rate by $1 \%$ [1] and so it is important to establish that the populations are indeed statistical.

The population of the atomic states will be described by statistical tensors as used by Nagamine and Yamazaki [5] and Kuno, Nagamine and Yamazaki [6] in their study of polarised muonic atoms. The statistical tensor $B_{k}(j), k=0,1 \ldots 2 j$ is proportional to the rank $k$ polarisation of the level $|j\rangle$ and is defined below. With this definition, $B_{0}(j)$ equals the population of the level $|j\rangle$.

$B_{k}(j)=\sqrt{2 j+1} \sum_{m}(-1)^{j-m} P_{m}\langle k 0 \mid j j m-m\rangle$

where $P_{m}$ is the population of the state $|j, m\rangle$. The conventions for the angular momentum algebra follow Brink and Satchler [7] throughout this letter. At atomic capture the atomic orbitals are filled without prejudice to $m_{l}$. This corresponds to the muons having negligible angular correlation with the beam direction [8]. After some fast internal Auger transitions 
the spin-orbit interaction splits terms according to $j$ and the statistical tensors for the level $n, l, j$ are,

$$
B_{k}(n, l, j)=\left[\frac{(2 j+1)^{3}}{(2 l+1)(2 s+1)}\right]^{\frac{1}{2}} \sum_{k_{1}, k_{2}} B_{k_{1}}(l) B_{k_{2}}(s)\left\langle k 0 \mid k_{1} k_{2} 00\right\rangle\left\{\begin{array}{ccc}
l & s & j \\
l & s & j \\
k_{1} & k_{2} & k
\end{array}\right\} .
$$

Only $B_{0}(l)$ is non-zero and using the triangular selection rule imposed by the ClebschGordan coefficient we see that only $B_{0}(n, l, j)$ and $B_{1}(n, l, j)$ are non-zero. This 'minimal polarisation' feature is a direct result of the isotropy of the muons just before atomic capture and the fact that muons are spin $\frac{1}{2}$ particles. $B_{1}(n, l, j)$ is proportional to $P_{\mu}$, the polarisation of the muon before atomic capture.

There follows an electromagnetic cascade whereby the muonic atom de-excites from $n \approx$ 14 to the $1 \mathrm{~S}$ level. For electric transitions of multipolarity $L$, the new statistical tensors due to the $n, l, j \rightarrow n^{\prime}, l^{\prime}, j^{\prime}$ transition are,

$$
\begin{aligned}
B_{k}\left(n^{\prime}, l^{\prime}, j^{\prime}\right) & =(2 l+1)\left(2 j^{\prime}+1\right) W\left(j j^{\prime} l l^{\prime} ; L \frac{1}{2}\right)^{2} u_{k}\left(j L j^{\prime}\right) B_{k}(n, l, j) \\
u_{k}\left(j L j^{\prime}\right) & =(-1)^{k+L-j-j^{\prime}}\left[(2 j+1)\left(2 j^{\prime}+1\right)\right]^{\frac{1}{2}} W\left(j j j^{\prime} j^{\prime} ; k L\right) .
\end{aligned}
$$

Thus, the statistical tensor of rank $k$ is fed only by the rank $k$ statistical tensors for higher levels. This feature may be termed 'selective feeding' since the tensor $B_{k}$ feeds other tensors according to the selection rule $\Delta k=0$. It follows that $B_{0}$ and $B_{1}$ will be the only non-zero statistical tensors during the cascade.

At the level where the hyperfine splitting becomes larger than the natural width, the statistical tensors for the states $|n, l, j, f\rangle$ are,

$B_{k}(n, l, j, f)=\left[\frac{(2 f+1)^{3}}{(2 i+1)(2 j+1)}\right]^{\frac{1}{2}} \sum_{k_{1}, k_{2}} B_{k_{1}}(i) B_{k_{2}}(n, l, j)\left\langle k 0 \mid k_{1} k_{2} 00\right\rangle\left\{\begin{array}{ccc}i & j & f \\ i & j & f \\ k_{1} & k_{2} & k\end{array}\right\}$,

where $B_{k_{1}}(i)$ is the statistical tensor for the nucleus. By observing the selection rule for $k$, $k_{1}$ and $k_{2}$, the total populations for the hyperfine states must have the following form, 


$$
B_{0}(n, l, j, f)=\alpha+\beta P_{\mu} P_{i}
$$

where $\alpha$ and $\beta$ are constants peculiar to the level $|n, l, j, f\rangle$ and $P_{i}$ is the vector polarisation of the nucleus. Higher rank polarisations of the nucleus cannot contribute to $B_{0}(n, l, j, f)$ since the rank of the total angular momentum polarisation is no higher than 1.

Allowing the hyperfine levels to decay only via electric transitions we have for the transition $n, l, j, f \rightarrow n^{\prime}, l^{\prime}, j^{\prime}, f^{\prime}$,

$$
\begin{aligned}
B_{k}\left(n^{\prime}, l^{\prime}, j^{\prime}, f^{\prime}\right)= & (2 l+1)\left(2 j^{\prime}+1\right) W\left(j j^{\prime} l l^{\prime} ; L \frac{1}{2}\right)^{2} \times(2 j+1)\left(2 f^{\prime}+1\right) W\left(f f^{\prime} j j^{\prime} ; L \frac{1}{2}\right)^{2} \\
& \times u_{k}\left(f L f^{\prime}\right) B_{k}(n, l, j, f)
\end{aligned}
$$

which has the same 'selective feeding' property as equation (3). Using this fact and equation (6) the $1 \mathrm{~S}$ hyperfine populations can be parameterised by,

$$
B_{0}\left(1,0, \frac{1}{2}, f\right)=\gamma+\delta P_{\mu} P_{i} .
$$

The parameter $\gamma$ may be found either by demanding that the populations are statistical when $P_{\mu}=P_{i}=0$ or by directly calculating it using equations (2) (3) (5) and (7).

$$
\gamma=\frac{2 f+1}{2(2 i+1)}
$$

Thus, if either $P_{\mu}=0$ or $P_{i}=0$, the total populations of the 1S hyperfine levels are statistical in the limit that no magnetic transitions occur during the cascade. This is due to minimal polarisation after atomic capture and selective feeding during the cascade.

\section{ACKNOWLEDGMENTS}

This research was supported by the British Royal Society under the auspices of the European Science Exchange Programme. 


\section{REFERENCES}

[1] N. C. Mukhopadhyay, Phys. Rep. 30, C1 (1977).

[2] D. Favart, F. Brouillard, L. Grenacs, P. Igo-Kemenes, P. Lipnik, and P. C. Macq, Phys. Rev. Lett. 25, 1348 (1970).

[3] J. Deutsch, private communication.

[4] J. G. Congleton and H. W. Fearing, Nucl. Phys. A, (1993) in press.

[5] K. Nagamine and T. Yamazaki, Nucl. Phys. A219, 104 (1974).

[6] Y. Kuno, K. Nagamine and T. Yamazaki, Nucl. Phys. A475 , 615 (1987).

[7] D. M. Brink and G. R. Satchler, Angular Momentum ( Clarendon Press, Oxford 1971 ) .

[8] R. A. Mann and M. E. Rose, Phys. Rev. 121, 293 (1961). 